\title{
Mean-field theory of the Potts gas
}

\author{
Hans-Otto Georgii ${ }^{1}$, Salvador Miracle-Sole ${ }^{2}$, Jean Ruiz ${ }^{2}$ and \\ Valentin A Zagrebnov ${ }^{3}$ \\ ${ }^{1}$ Mathematisches Institut der Universität München, Theresienstr. 39, D-80333 München, \\ Germany \\ 2 Centre de Physique Théorique, CNRS, UMR 6207, Luminy Case 907, \\ F-13288 Marseille Cedex 9, France \\ ${ }^{3}$ Université de la Méditerranée and Centre de Physique Théorique, UMR 6207, \\ Luminy Case 907, F-13288 Marseille Cedex 9, France \\ E-mail: georgii@math.lmu.de,miracle@cpt.univ-mrs.fr, ruiz@cpt.univ-mrs.fr and \\ zagrebnov@cpt.univ-mrs.fr
}

Received 20 February 2006, in final form 18 May 2006

Published 5 July 2006

Online at stacks.iop.org/JPhysA/39/9045

\begin{abstract}
We consider a gas of classical particles in $\mathbb{R}^{d}$ having $q$ distinct colours, interacting via a mean-field Potts potential and subject to an external field; a colour-independent molecular interaction of a mean-field type is also admitted. In contrast to the usual lattice Potts model, the Potts gas exhibits the specific volume $v$ as a parameter, in addition to colour-ordering. The $v$-dependence of the Potts gas is studied in detail, and the complete phase diagram is derived. It turns out that, for $q \geqslant 3$, the transition to colour-ordering implies a jump of density. For $q=2$, this transition is continuous but may become discontinuous under the influence of a suitable molecular interaction.
\end{abstract}

PACS numbers: $05.20 . J j$, 05.70.Fh

\section{Introduction}

Since its introduction in [1] in 1952, the Potts model on various lattices has intensively been studied, and a lot of rigorous results are available. In particular, the phase diagram of the Potts model on a complete graph, the so-called Curie-Weiss-Potts model, is completely understood $[2,3]$. For regular $d$-dimensional lattices, the Potts model has been shown to exhibit a temperature-driven first-order phase transition, provided the dimension $d$ is at least two and the number $q$ of states is large enough; see [4-10], the review paper [11] and the references therein. By way of contrast, much less is known for its continuous counterpart, the Potts gas of coloured point particles living in continuous space $\mathbb{R}^{d}$. The first model of this type was introduced by Widom and Rowlinson [12], having $q=2$ colours and hard-core intercolour repulsion. A colour-ordering transition for large activity has been established for this model 
by Ruelle [13], and for its soft-core counterpart by Lebowitz and Lieb [14]; see also the later studies by Bricmont, Kuroda and Lebowitz [5], Chayes, Chayes and Kotecky [15] and Georgii and Häggström [16]. No rigorous results, however, are available on the system's behaviour at the activity threshold to colour-ordering-in fact, even the existence of such a threshold is unknown — and only numerical results are available [17-19]. On the other hand, for a number of lattice models it has recently been shown that an understanding of the mean-field theory may be helpful for proving first-order phase transitions for real systems [20, 21]. So, it might also be worthwhile to clarify the mean-field theory of the Potts gas as a first step in understanding the behaviour of the real Potts gas at the activity threshold. This is the object of the present study.

We now describe the Potts gas in more detail. Consider a system of point particles sitting randomly inside a box $\Lambda$, of volume $|\Lambda|$, in the space $\mathbb{R}^{d}$. Each particle may have one of $q \geqslant 2$ different types, or colours. Just as in the lattice Potts model, we assume that particles of different colours repel each other. The position of particle $i$ is denoted by $\xi_{i} \in \Lambda$, its colour by $\sigma_{i}=1, \ldots, q$, and the total number of particles by $N$. The true Potts gas is then characterized by its potential energy

$H=\frac{1}{2} \sum_{i, j=1, \ldots, N} J\left(\xi_{i}-\xi_{j}\right)\left(1-\delta_{\sigma_{i}, \sigma_{j}}\right)-h \sum_{i=1, \ldots, N} \delta_{1, \sigma_{i}}+\frac{1}{2} \sum_{i, j=1, \ldots, N} \phi\left(\xi_{i}-\xi_{j}\right)$.

Here, $J(\cdot)$ is a positive function describing the strength of intercolour-repulsion, and $\delta_{\sigma_{i}, \sigma_{j}}$, the Kronecker symbol, is equal to 1 when $\sigma_{i}=\sigma_{j}$, and 0 otherwise. The first term thus represents a Potts interaction of colours. The second term describes the effect of a uniform external field acting on particles of colour 1, and the third corresponds to a colour-independent two-body interaction between the particles. Here, however, we shall study only the mean-field counterpart of the true Potts gas, which is obtained by replacing $H$ with

$$
H_{\Lambda, N}=\frac{J}{2|\Lambda|} \sum_{i, j=1, \ldots, N}\left(1-\delta_{\sigma_{i}, \sigma_{j}}\right)-h \sum_{i=1, \ldots, N} \delta_{1, \sigma_{i}}+N u\left(\frac{|\Lambda|}{N}\right),
$$

where $J>0$ is a position-independent interaction between all pairs of particles and $u$ is a function of the specific volume $v=|\Lambda| / N$ describing a molecular interaction that is independent of colour and position. For example, the case $u(v)=1 / v$ corresponds to a molecular pair repulsion of order $1 /|\Lambda|$. For $q=2$, a Hamiltonian of type (1) has been used in [22] to describe ferrofluid models. Note that the particle number $N$ or, equivalently, the specific volume $v$, is considered as a free variable of our model, and the Hamiltonian per volume $H_{\Lambda, N} /|\Lambda|$ has order $1 / v$. This $v$-dependence is an essential ingredient of the Potts gas which is not present in the lattice case, and it is our main object of study.

In section 2 we derive a variational formula for the Helmholtz free energy per particle. Then, in section 3, we solve this formula and compute the pressure in the case of a pure colour interaction and no external field, i.e., when $h=0$ and $u \equiv 0$. In section 4 we deal with the case of a non-vanishing external field $h$, and in section 5 we discuss the effect of a molecular background interaction $u$.

\section{Some thermodynamics}

The canonical partition function for the Hamiltonian (1) in a box $\Lambda$ at inverse temperature $\beta=1 / k_{B} T$ reads

$$
Z_{\text {can }}(\Lambda, N, \beta)=\frac{1}{N ! \lambda^{N d}} \int_{\Lambda} \mathrm{d} \xi_{1} \cdots \int_{\Lambda} \mathrm{d} \xi_{N} \sum_{\sigma_{1} \cdots \sigma_{N}} \mathrm{e}^{-\beta H_{\Lambda, N}},
$$


the quantity $\lambda=\left(2 \pi \hbar^{2} \beta / m\right)^{1 / 2}$ takes care of the kinetic energy of particles. The Helmholtz free energy per particle is then

$$
f(v, \beta)=-\frac{1}{\beta} \lim _{N \rightarrow \infty} \frac{1}{N} \ln Z_{\mathrm{can}}(\Lambda, N, \beta),
$$

where the limit is taken in such a way that $|\Lambda| / N$ tends to the specific volume $v>0$. According to standard thermodynamic formalism, the corresponding canonical pressure as a function of $v$ and $\beta$ is given by the derivative

$$
p(v, \beta)=-\frac{\partial}{\partial v} f(v, \beta),
$$

provided this derivative exists. The above function $f(v, \beta)$, however, is in general not convex in $v$, and $p(\cdot, \beta)$ is not decreasing. The true thermodynamic free energy is therefore given by the convex envelope of the $f(\cdot, \beta)$, and the true thermodynamic pressure by the negative derivative of this convex envelope, which amounts to applying Maxwell's construction to $p(\cdot, \beta)$.

We also introduce a microcanonical partition function, in which the sum in (2) is restricted to the configurations $\left(\sigma_{1}, \ldots, \sigma_{N}\right)$ such that, for each $a=1, \ldots, q$, the number $N_{a}$ of particles of colour $a$ is fixed. This partition function reads

$$
Z_{\text {micr }}\left(\Lambda,\left\{N_{a}\right\}, \beta\right)=\frac{|\Lambda|^{N}}{N ! \lambda^{N d}} \frac{N !}{N_{1} ! \cdots N_{q} !} \mathrm{e}^{-\beta H_{\Lambda, N}\left(\left\{N_{a}\right\}\right)},
$$

where $H_{\Lambda, N}\left(\left\{N_{a}\right\}\right)$ is the energy (1) for such configurations. In fact, setting $J=1$ and replacing $\beta h$ by $h$ and $\beta u$ by $u$ we have

$$
\beta H_{\Lambda, N}\left(\left\{N_{a}\right\}\right)=\frac{\beta}{2|\Lambda|}\left(N^{2}-\sum_{a=1}^{q} N_{a}^{2}\right)-h N_{1}+N u(|\Lambda| / N) .
$$

The corresponding free energy per particle is

$$
\varphi\left(v,\left\{x_{a}\right\}, \beta\right)=-\frac{1}{\beta} \lim _{N \rightarrow \infty} \frac{1}{N} \ln Z_{\text {micr }}\left(\Lambda,\left\{N_{a}\right\}, \beta\right),
$$

where $\left\{x_{a}\right\}_{a=1}^{q}$ are positive numbers satisfying $x_{1}+\cdots+x_{q}=1$, and the limit is taken in such a way that $N_{a} / N \rightarrow x_{a}$ for $a=1, \ldots, q$. The usual argument proving equivalence of ensembles then tells us that

$$
f(v, \beta)=\min _{\left\{x_{a}\right\}} \varphi\left(v,\left\{x_{a}\right\}, \beta\right) .
$$

To compute $\varphi$ we use Stirling's formula, showing that

$$
\begin{aligned}
& |\Lambda|^{N} / N ! \sim \exp N(\ln v+1) \\
& \frac{N !}{N_{1} ! \cdots N_{q} !} \sim \exp \left(-N \sum_{a=1}^{q} x_{a} \ln x_{a}\right)
\end{aligned}
$$

at the leading order in $N$. It thus follows from equation (6) that

$\beta \varphi\left(v,\left\{x_{a}\right\}, \beta\right)=-\ln \left(v / \lambda^{d}\right)-1+u(v)+\sum_{a=1}^{q} x_{a} \ln x_{a}+\frac{\beta}{2 v}\left(1-\sum_{a=1}^{q} x_{a}^{2}\right)-h x_{1}$.

Note that the next to last term, the Potts interaction term, combines the variables $v$ and $\left\{x_{a}\right\}$. But to compute the minimum (7), for given $\beta$ and $v$, we only need to minimize the function

$$
F\left(\left\{x_{a}\right\}\right)=\sum_{a=1}^{q} x_{a} \ln x_{a}+\frac{\beta}{2 v}\left(1-\sum_{a=1}^{q} x_{a}^{2}\right)-h x_{1}
$$


over the parameters $\left\{x_{a}\right\}$, and this minimization problem is well known from the analysis of the usual mean-field Potts model on the lattice: the case $h=0$ was discussed by Wu in [2], and the case $h \neq 0$ has recently been studied by Biskup et al [20, 21] (see also [10]). In the next sections we collect their results and discuss the consequences for the $v$-dependence of $f(v, \beta)$ and $p(v, \beta)$.

\section{The case of no external field}

Let us first consider the case $h=0$. In this case the minima of the function $F$ in equation (9) can be parametrized by a real number $s, 0 \leqslant s \leqslant 1$, such that the minimizing vectors $\left(x_{1}, \ldots, x_{q}\right)$ have the components

$$
\frac{1+(q-1) s}{q}, \frac{1-s}{q}, \ldots, \frac{1-s}{q}
$$

in arbitrary order. Moreover, there exists a threshold value $\beta_{0}^{(q)}$, given by

$$
\beta_{0}^{(2)}=2 \quad \text { and } \quad \beta_{0}^{(q)}=2 \frac{q-1}{q-2} \ln (q-1) \quad \text { for } \quad q \geqslant 3,
$$

such that the following holds:

(1) For $\beta / v<\beta_{0}^{(q)}$, the function $F\left(\left\{x_{a}\right\}\right)$ has the unique minimizer $x_{1}=\cdots=x_{q}=1 / q$, i.e., the minimum is attained at $s=0$.

(2) In the case $\beta / v>\beta_{0}^{(q)}$, the function $F\left(\left\{x_{a}\right\}\right)$ has $q$ distinct minimizers, corresponding to $q$ permutations of the components (10). The minimum is attained at $s=s_{0}>0$, where $s_{0}$ is the largest solution of the equation

$$
\frac{1}{s} \ln \frac{1+(q-1) s}{1-s}=\frac{\beta}{v} \text {. }
$$

Note that, for the vectors of the form (10), the function $F$ takes the form

$$
F(s)=\frac{1+(q-1) s}{q} \ln \frac{1+(q-1) s}{q}+(q-1) \frac{1-s}{q} \ln \frac{1-s}{q}+\frac{\beta}{2 v} \frac{q-1}{q}\left(1-s^{2}\right) .
$$

In case 1 we have $F(0)<F(s)$ for all $0<s \leqslant 1$. In case 2 one gets $F(0)>F\left(s_{0}\right)$, where $s_{0}$ is the local minimizer of $F$ in the unit interval; equation (12) corresponds to the condition $\mathrm{d} F /\left.\mathrm{d} s\right|_{s=s_{0}}=0$.

Collecting the preceding observations and taking equations (7) and (8) into account we find that, for $h=0$ and $u \equiv 0$, the free energy in (3) can be expressed as

$$
f(v, \beta)=-\beta^{-1}\left(1+\ln \left(v / \lambda^{d}\right)+\min _{s} F(s)\right),
$$

that is,

$$
f(v, \beta)= \begin{cases}-\beta^{-1}\left(1+\ln \left(v / \lambda^{d}\right)+F(0)\right) & \text { if } v>\beta / \beta_{0}^{(q)}, \\ -\beta^{-1}\left(1+\ln \left(v / \lambda^{d}\right)+F\left(s_{0}\right)\right) & \text { if } v<\beta / \beta_{0}^{(q)} .\end{cases}
$$

Here, in the first case, $F(0)=-\ln q+\beta(q-1) /(2 q v)$, whereas in the second case the argument $s_{0}=s_{0}(\beta / v)$ is a function of $\beta / v$ defined by equation (12). Since by (12) one has $\mathrm{d} s_{0} / \mathrm{d} v=0$, differentiating of (13) with respect to the specific volume $v$ gives for the pressure

$$
p(v, \beta)= \begin{cases}1 /(\beta v)+(q-1) /\left(2 q v^{2}\right) & \text { if } v>\beta / \beta_{0}^{(q)} \\ 1 /(\beta v)+\left((q-1) /\left(2 q v^{2}\right)\right)\left(1-s_{0}^{2}\right) & \text { if } v<\beta / \beta_{0}^{(q)}\end{cases}
$$



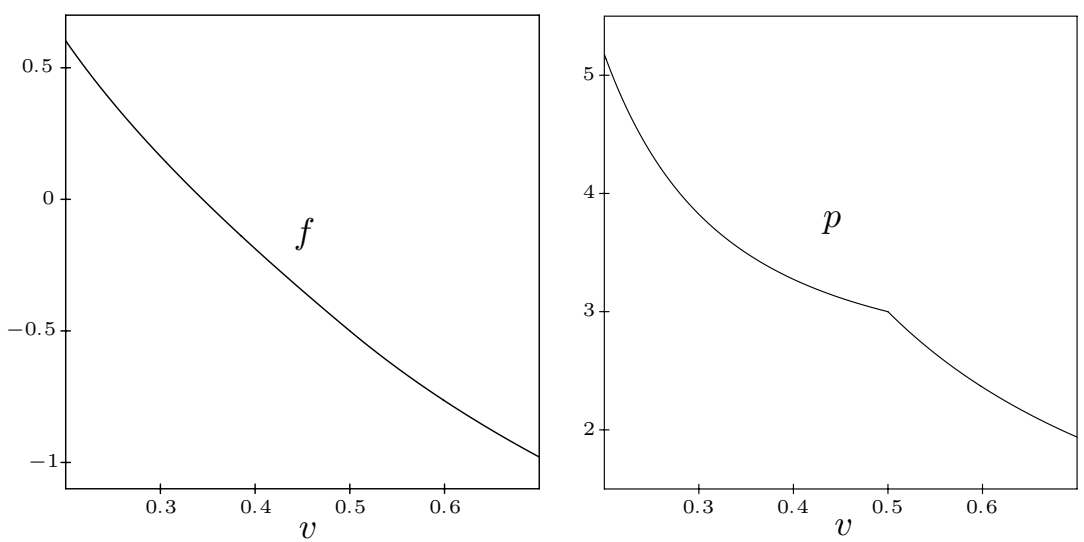

Figure 1. The free energy $f$ and the pressure $p$ as functions of the specific volume $v$ in the Ising case $q=2$ with $\beta=\lambda=1, h=0$ and $u \equiv 0$.

These considerations are valid for any $q \geqslant 2$. However, the behaviour at the critical value $v_{0}=\beta / \beta_{0}^{(q)}$ is different for $q=2$ (case of Ising spins) and $q \geqslant 3$. Note that, for $v=v_{0}$, the solution of equation (12) is $s_{0}=(q-2) /(q-1)$, which is zero for $q=2$ and non-zero for $q \geqslant 3$. Consequently,

(1) if $q=2$ and $v=v_{0}:=\beta / 2$, the minimum is attained at $s=0$ and there is only one minimizer of $F$;

(2) if $q \geqslant 3$ and $v=v_{0}:=\beta / \beta_{0}^{(q)}$, the minimum is attained at the two distinct values $s=0$ and $s=s_{0}:=(q-2) /(q-1)$. There are thus $q+1$ minimizers of $F$.

Since $F(0)=F\left(s_{0}\left(\beta / v_{0}\right)\right)$ is the minimal value of $F$, the free energy function $f(v, \beta)$ in (13) is always continuous in $v$. For $q=2$, its derivative $p(v, \beta)$ defined by (14) is also continuous in $v$ because $s_{0}\left(\beta / v_{0}\right)=0$. In fact, $p(\cdot, \beta)$ is decreasing and $f(\cdot, \beta)$ is convex (see figure 1 ).

For $q \geqslant 3$, however, the above observations yield that $p(v, \beta)$ exhibits a positive jump at the point $v_{0}=\beta / \beta_{0}^{(q)}$, for any $\beta$. Specifically,

$$
p\left(v_{0}+0, \beta\right)-p\left(v_{0}-0, \beta\right)=(q-2)^{2} /\left(2 q(q-1) v_{0}^{2}\right)>0 .
$$

Since $p(v, \beta)$ tends to $+\infty$ when $v \rightarrow 0$ and to 0 when $v \rightarrow \infty$, this implies that the monotonicity of $p(v, \beta)$ in $v$ is broken. Equivalently, the free energy function $f(v, \beta)$ is not a convex function of $v$. The situation is thus analogous to that of the isotherms at low temperature of the classical van der Waals theory. This means that a phase transition exists, at a certain value of the pressure, between a dense and a dilute phase. This transition can be determined by the standard Maxwell's construction (see figure 2).

We also notice that in order to draw the different isotherms for given values of $\beta$, i.e., the curves associated with the pressure $p(v, \beta)$ as a function of $v$, we can use a simple parametric representation for the branches $v<\beta / \beta_{0}^{(q)}$. This representation is defined by the following mapping:

$$
s \rightarrow(v(s), p(s)), \quad \text { for } \quad(q-2) /(q-1) \leqslant s \leqslant 1,
$$

where the functions $v(s)$ and $p(s)$ are given, respectively, by equations (12) and (14). The analogous parametric representation for the free energy $f(v, \beta)$ is given by equations (12) and (13). 

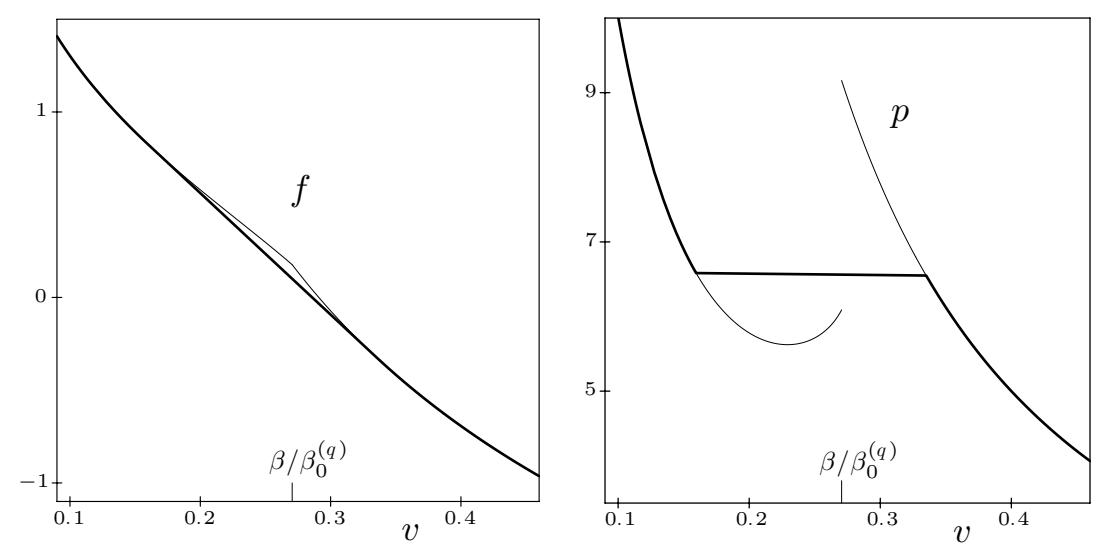

Figure 2. The free energy $f$ (thin) with its convex envelope (bold) and the pressure $p$ (thin) with the corresponding Maxwell's construction (bold) as functions of the specific volume $v$ for the parameters $q=5, \beta=\lambda=1, h=0$ and $u \equiv 0$.

\section{Including an external field}

Next we discuss the case $h \neq 0$, but still with $u \equiv 0$. For given $\beta, v$ and $h$, any minimizer $\left(x_{1}, \ldots, x_{q}\right)$ of the function $F\left(\left\{x_{a}\right\}\right)$ in (9) then has the following properties (see [21]):

(1) For $h>0$,

$$
x_{1}>x_{2}=\cdots=x_{q} .
$$

(2) For $h<0$, all minimizers are permutations in the last $q-1$ variables of vectors satisfying

$$
x_{1}<x_{2}=\cdots=x_{q-1} \leqslant x_{q} .
$$

Considering (9) for $h \neq 0$ one finds in analogy to (11) that for $q \geqslant 3$ there exists a continuous, strictly positive, strictly decreasing function $\beta_{0}^{(q)}(h)$ on an interval $\left(0, h_{c}\right)$, where $h_{c}:=\ln q-2(q-2) / q$ is a critical field. For $q \geqslant 4$, this function is also defined for all $h<0$, and is strictly increasing there. The function $\beta_{0}^{(q)}(h)$ therefore determines the phase diagram of the system. It takes the value $\beta_{0}^{(q)}(0)=2((q-1) /(q-2)) \ln (q-1)$ at $h=0$, and has the limiting values $\beta_{0}^{(q-1)}$ when $h \rightarrow-\infty$ and $4(q-1) / q$ when $h \rightarrow h_{c}$ (see figure 3$)$. The case 1 above $(q \geqslant 3, h>0)$ thus splits into the following subcases:

(1.1) For all $(v, h)$ such that either $h \geqslant h_{c}$ or $v \neq v_{0}(h):=\beta / \beta_{0}^{(q)}(h), h>0$, there is a unique minimizer of the function $F$. This minimizer satisfies condition (15).

(1.2) If $0<h<h_{c}$ and $v=v_{0}(h)$, there exist two minimizers both satisfying (15).

On the other hand, for $h<0$ and $q \geqslant 4$ we obtain the subcases

(2.1) If $h<0$ and $v>v_{0}(h)$, there exists a unique minimizer of $F$, and is such that $x_{1}<x_{2}=\cdots=x_{q}$.

(2.2) If $h<0$ and $v<v_{0}(h)$, there are $q-1$ distinct minimizers, corresponding to permutations of the last $q-1$ components of a vector satisfying condition (16).

(2.3) For $h<0$ and $v=v_{0}(h)$ there exist $q$ minimizers, one of them as in case 2.1, and the other $q-1$ as in case 2.2 . 


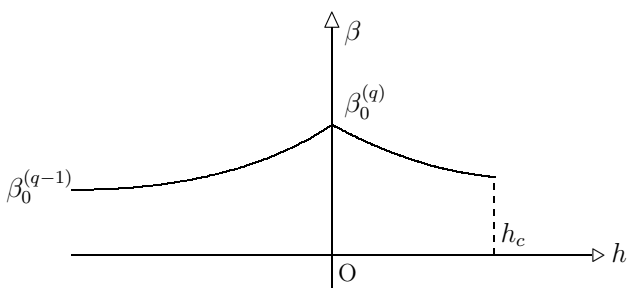

Figure 3. The phase diagram for $q \geqslant 4$, defined by the graph of $\beta_{0}^{(q)}(h)$.

Consider now the cases 1.1 and 1.2 in more detail. Just as in the case of zero field, the minimizers of $F$ can be parametrized by $0 \leqslant s \leqslant 1$ (see (10)). According to (15), the component $x_{1}$ in the representation (10) is always the largest one, and permutations play no role. By virtue of (9) and (10) the value of the parameter $s=s_{0}(h, \beta / v)$ corresponding to a minimizer is defined by the equation

$$
\frac{1}{s}\left(\ln \frac{1+(q-1) s}{1-s}-h\right)=\frac{\beta}{v} .
$$

In case 1.1 , this equation has a unique solution $s_{0}$ in the interval $(0,1)$. In case 1.2 one gets two minimizers; the corresponding values of $s_{0}$ are given by the smaller and the larger solution of equation (17). It follows that the analysis of case $1.2\left(0<h<h_{c}\right)$ is very similar to the one we carried out for $h=0$ in section 3. For given $\beta$ and $h$ in the interval $\left(0, h_{c}\right)$, the parameter $s_{0}(h, \beta / v)$ has, as a function of $v$, a discontinuity at the point $v=v_{0}(h)$. This implies that $p(v, \beta, h)$, as a function of $v$, has also a discontinuity at this point. Therefore, the same arguments as in section 3 allow us to conclude that in the presence of a non-zero field $0<h<h_{c}$ the system still exhibits a phase transition of first order from a dense to a dilute phase. For $h \geqslant h_{c}$, however, $p(v, \beta, h)$ is continuous in $v$. On the other hand, if one fixes $\beta$ and $v$ such that $4(q-1) / q<\beta / v \leqslant \beta_{0}^{(q)}$ and varies $h$, then the parameter $s_{0}(h, \beta / v)$, and hence also $p(\beta, v, h)$, shows a discontinuity at the point $h=h_{0}$ solving $v / \beta=\beta_{0}^{(q)}\left(h_{0}\right)$. That is, for such $\beta$ and $v$, the system also undergoes a discontinuous transition from a dense to a dilute phase when the external field $h$ is varied. Finally, just as at the end of section 3 we observe that using equation (17) to represent $v$ or $h$ in terms of $s$ one obtains a convenient parametric representation of the functions $f(v, \beta, h)$ and $p(v, \beta, h)$ in dependence of $v$ or $h$.

In the alternate cases 2.1-2.3 when $h<0$, the discussion is more complicated and no explicit formulae are available. For given $h$, the system then behaves, in some sense, as a Potts model with $q-1$ colours. Since under the conditions of case 2.3 there exist $q$ distinct minimizers of $F$, the pressure $p(v, \beta, h)$ then takes two different values (because permutations of the $\left\{x_{a}\right\}$ do not affect the value of $p$ ). Hence $p$ has a discontinuity at the point $v_{0}(h)$. Arguing as above, we conclude that, for fixed $\beta$ and $h<0$ and varying $v$, the system undergoes a discontinuous transition from a dense to a dilute phase, and similarly for fixed $\beta / v$ in the interval $\left(\beta_{0}^{(q-1)}, \beta_{0}^{(q)}\right)$ and varying $h$; see figure 3 .

\section{Adding a molecular interaction}

Finally we turn to the case when the system is also subject to a background potential describing a colour-independent intermolecular interaction of particles; such an interaction leads to the third term of $H_{\Lambda, N}$ in equation (1). As noticed above, such Hamiltonians have been used for $q=2$ and $h=0$ in [22] to describe ferrofluid models. To make contact with that paper we 

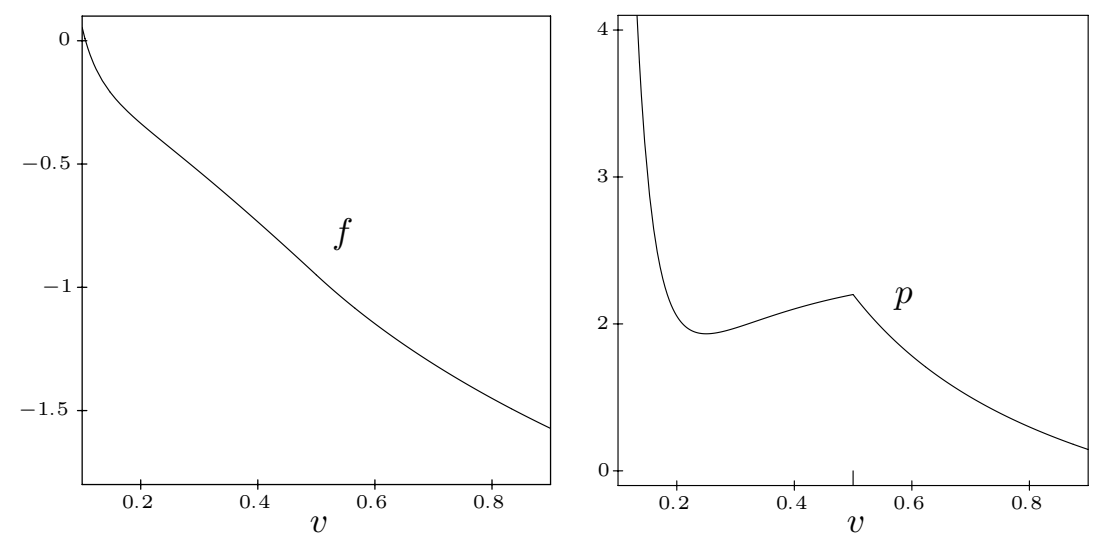

Figure 4. Free energy $f$ and pressure $p$ for $q=2, \beta=\lambda=1, h=0$, and the molecular potential $u(v)=-1 /(4 v)+1 /\left(80 v^{2}\right)$, the mean-field counterpart of a two-body attraction and a three-body repulsion; the convex hull of $f$ and Maxwell's construction for $p$ are omitted.

note that, in the Ising case when $q=2$ and $\sigma_{i}= \pm 1$, one has $1-\delta_{\sigma_{i}, \sigma_{j}}=\left(1-\sigma_{i} \sigma_{j}\right) / 2$. The Hamiltonian of equation (1) thus coincides with that of ([22] equation (2)) as soon as one sets in (1) $q=2, h=0, \beta=1$,

$$
u(v)=v g\left(\frac{1}{v}\right)-\frac{J}{2 v},
$$

and replaces $J$ by $J / 2$, where $g$ is as in [22]. In this reference, it was assumed that $g$ is smooth on $\mathbb{R}_{+}, \lim _{\rho \downarrow 0} g^{\prime \prime}(\rho)$ exists and $\lim _{\rho \downarrow \infty} g^{\prime \prime}(\rho)=\infty$. The last condition was imposed to ensure the thermodynamic stability for all choices of $J$. In the present setting of equation (1), this can simply be achieved by requiring that $u \geqslant 0$.

According to [22], for different choices of $g$ one gets various different scenarios of phase transition that manifest a positive feedback of the magnetization to the particle density. In particular, one finds phase diagrams showing both second-order transitions as well as firstorder transitions with jumps of density and magnetization. As we have seen in sections 3 and 4 , for $q \geqslant 3$, such jumps do exist even without any molecular interaction.

To deal with the model equation (1) with non-zero $u$ it is sufficient to note that the term $u(v)$ in (8) does not depend on the densities $\left\{x_{a}\right\}$. The previous analysis of the minimizers of the free energy can thus be applied. We find that, for $h=0$, the term $u(v)$ simply has to be added to the expressions on the right-hand side of (13), and $-u^{\prime}(v)$ has to be added to the right-hand side of (14). Consequently, the discontinuity of $p(\cdot, \beta, h)$ at $v_{0}=\beta / \beta_{0}^{(q)}(h)$ for $q \geqslant 3$, found in sections 3 and 4 , still persists when any continuous molecular contribution $u \geqslant 0$ is added. On the other hand, the continuous transition for $q=2$ and $u \equiv 0$ can be turned into a discontinuous transition by adding a molecular contribution $u$ with a sufficiently strong non-convexity that beats the convexity of the right-hand side of (13); see figure 4 and the discussion in [22].

\section{Acknowledgments}

This work was begun during H-OG's visits to the Centre de Physique Théorique, Luminy, UMR 6207, Université de Provence, Aix-Marseille I; Université de la Méditerranée, AixMarseille II; Université du Sud, Toulon, Var; FRUMAM (FR 2291) and CNRS. He gratefully 
acknowledges the warm hospitality of CPT and the financial support of the Université du Sud, Toulon, Var.

\section{References}

[1] Potts R B 1952 Some generalized order-disorder transitions Proc. Camb. Phil. Soc. 48 106-9

[2] Wu F Y 1982 The Potts model Rev. Mod. Phys. 54 235-68

[3] Costenic M, Ellis R and Touchette H 2005 Complete analysis of phase transitions and ensemble equivalence for the Curie-Weiss-Potts model J. Math. Phys. 46063301

[4] Kotecký R and Shlosman S 1982 First-order phase transitions in large entropy lattice systems Commun. Math. Phys. 83 493-515

[5] Bricmont J, Kuroda K and Lebowitz J L 1985 First-order phase transitions in lattice and continuous systems Commun. Math. Phys. 101 501-38

[6] Laanait L, Messager A and Ruiz J 1986 Phase coexistence and surface tension for the Potts model Commun. Math. Phys. 105 527-45

[7] Martirosian D H 1986 Translation invariant Gibbs states in the $q$ states Potts model Commun. Math. Phys. 105 281-90

[8] Kotecký R, Laanait L, Messager A and Ruiz J 1990 The $q$-state Potts model in the standard Pirogov-Sinai theory: surface tension and Wilson loops J. Stat. Phys. 58 199-248

[9] Bakchich A, Benyoussef A and Laanait L 1989 Phase diagram of the Potts model in an external magnetic field Ann. Inst. H Poincaré Phys. Théor. 50 17-35

[10] Bollobas B, Grimmett G and Janson S 1996 The random-cluster model on the complete graph Probab. Theory Rel. Fields $104283-317$

[11] Georgii H-O, Häggström O and Maes C 2000 The random geometry of equilibrium phases Phase Transitions and Critical Phenomena vol 18, ed C Domb and J L Lebowitz (New York: Academic) pp 1-142

[12] Widom B and Rowlinson J S 1970 New model for the study of liquid-vapor phase transition J. Chem. Phys. 52 1670-84

[13] Ruelle D 1971 Existence of phase transition in a continuous classical system Phys. Rev. Lett. 27 1040-1

[14] Lebowitz J L and Lieb H E 1972 Phase transition in a continuum classical system with finite interactions Phys. Lett. A 39 98-100

[15] Chayes J T, Chayes L and Kotecký R 1995 The analysis of the Widom-Rowlinson model by stochastic geometric methods Commun. Math. Phys. 172 551-69

[16] Georgii H-O and Häggström O 1996 Phase transition in continuum Potts models Commun. Math. Phys. $181507-28$

[17] Johnson G, Gould H, Machta J and Chayes L K 1997 Monte Carlo study of the Widom-Rowlinson fluid using cluster methods Phys. Rev. Lett. $792612-5$

[18] Sun R, Gould H, Machta J and Chayes L W 2000 Cluster Monte Carlo study of multicomponent fluids of the Stillinger-Helfand and Widom-Rowlinson type Phys. Rev. E 62 2226-32

[19] Georgii H-O, Lörinczi J and Lukkarinen J The continuum Potts model at the disorder-order transition-a study by cluster dynamics J. Stat. Mech. 2005 P06011

[20] Biskup M and Chayes L 2003 Rigorous analysis of discontinuous phase transitions via mean-field bounds Commun. Math. Phys. 238 53-93

[21] Biskup M, Chayes L and Crawford N 2006 Mean-field driven first-order phase transitions in systems with long-range interactions J. Stat. Phys. 122 1139-93

[22] Georgii H-O and Zagrebnov V 1998 On the interplay of magnetic and molecular forces in Curie-Weiss ferrofluid models J. Stat. Phys. 93 79-107 